\title{
A Modified Random Phase Approximation of Polyelectrolyte Solutions
}

\author{
A. V. Ermoshkin, M. Olvera de la Cruz \\ Department of Materials Science and Engineering, \\ Northwestern University, Evanston, IL, USA 60208-3108
}

(October 5, 2018)

\begin{abstract}
We compute the phase diagram of salt-free polyelectrolyte solutions using a modified Debye-Huckel Approach. We introduce the chain connectivity via the Random Phase Approximation with two important modifications. We modify the electrostatic potential at short distances to include a bound on the electrostatic attractions at the distance of closest approach between charges. This modification is shown to act as a hard core in the phase diagram of electrolyte solutions. We also introduce a cut-off on the integration of the modes of wave length smaller than the size over which the chains are strongly perturbed by the electrostatic interactions. This cut-off is shown to be essential to predict physical phase diagram in long chain solutions.
\end{abstract}

\section{INTRODUCTION}

Debye-Hückel (DH) theory derived nearly 80 years ago successfully explains many thermodynamic properties of dilute symmetric electrolyte solutions. ${ }^{1}$ Recent computer simulations of hard-sphere ionic fluids ${ }^{2}$ show that the DH approach for finite size ion solutions provides a rather accurate estimate of the critical temperature, although it underestimates the critical density. A conceptually simple modification of the DH approach, based on accounting for the interactions of ionic pairs introduced by Bjerrum $^{3}$ with residual ionic fluid, however, gives better estimates for the critical parameters. ${ }^{4}$

Though a large scientific effort has been made towards understanding the thermodynamics of simple electrolyte solutions, flexible charged chains solutions are not understood. The Random Phase Approximation (RPA), a $\mathrm{DH}$ approach which includes the structure function of the chains, is widely used to describe polyelectrolytes solutions. ${ }^{5,6}$ RPA, however, provides unphysical coexisting curves in salt free polyelectrolyte solutions. ${ }^{7}$ In particular, RPA predicts that the critical density decreases with increasing the chains degrees of polymerization $N$, and it goes to zero as $N$ goes to infinite. Moreover, the critical temperature increases rapidly as $N$ increases suggesting that strongly charged chains are not water soluble in salt-free solutions. Recent computer simulations of charged chains reveal a critical density rather insensitive to the value of $N$, and a critical temperature that does increases as $N$ increases but not as rapidly as predicted by the RPA approach. ${ }^{8}$ A one component plasma approach to polyelectrolytes solutions gives both a critical density and a critical temperature insensitive to the the degree of polymerization $N .^{9}$ The one component plasma approach ignores the contribution from the fluctuations of the monomers. That is, the one component plasma assumes that the chains provide a constant charge density support over which the counterions fluctuate. It is difficult to demonstrate and understand why and when it is possible to neglect the chain density fluctuations. Moreover, the value of the critical temperature is strongly un- derestimated in the one component plasma approach to polyelectrolytes salt-free solutions.

Here we describe a modified polyelectrolyte RPA model that predicts realistic values of the critical temperature, and a critical density rather insensitive to $N$ in agreement with the simulations. The RPA approach described here includes two modifications. The first modification is related to the cut-off in RPA. In salt free polyelectrolyte solution the electrostatic contribution to the free energy of charged Gaussian chains does not reduces to the DH limiting law. ${ }^{5}$ Instead, a more strong electrostatic contribution results in salt-free solutions that generates instabilities in the free energy as the monomer concentration goes to zero at room temperatures in strongly charged chains. ${ }^{7}$ Scaling arguments ${ }^{10-12}$ and computer simulations, ${ }^{13,14}$ however, show that charged chains are stretched in monovalent ionic solutions on length scales smaller and of the order of the inverse screening length. Therefore, it is not possible to include the monomer density fluctuations within RPA, which assumes that the electrostatic interactions do not perturbed the chain statistics (a linear response theory). Here we introduce a concentration dependent cut off proportional to the inversive screening length in the RPA electrostatic contribution to include only the contribution from the unperturbed Gaussian chains. We also discuss other possibilities for the cut-off that yield similar modifications in the phase diagram. The second and less important modification involves including the contribution from the hard core of the ions and the monomers. In simple electrolyte solutions the hard core of the ions is included in the electrostatic contribution to the free energy to avoid spurious results as the concentration of ions increases. In this paper we modify the interaction potential and show that our modified potential describes the polyelectrolyte solution at the same level of approximation as the modified DH for hard-sphere ionic fluids.

In Section II we describe the modified RPA model for salt-free flexible strongly charged chains. In Section III we discuss the electrostatic interaction potential used in the model. In Section IV we state the problem of RPA 
describing Gaussian chains and the required modifications. In Section $\mathrm{V}$ we outline and discuss the results, and give some conclusions.

\section{MODEL AND FREE ENERGY}

Let us consider a mixture of negatively charged polyelectrolyte chains and a residual ionic fluid. We assume that every chain consists of $N$ monomers each carrying a negative charged $-q$ and we denote the concentration of monomers as $\rho_{-}$. Every ion in the system carries the charge $+q$ and their total concentration is $\rho_{+}$. Due to electroneutrality the following condition holds

$$
\rho_{-}=\rho_{+}
$$

We write free energy of the system in the form

$$
F=F_{\text {ref }}+F_{\text {el }}
$$

Here $F_{\text {ref }}$ is the free energy of the system without any electrostatic interactions and in the limit of good solvent it could be written as

$$
\frac{F_{\text {ref }}}{T V}=\rho_{+} \ln \rho_{+}+\frac{\rho_{-}}{N} \ln \rho_{-}+\frac{1}{a^{3}}\left(1-\rho^{*}\right) \ln \left(1-\rho^{*}\right)
$$

where $T$ and $V$ is the temperature (in units of Boltzmann constant $k_{B}$ ) and the volume of the system respectively. We also assumed that monomers of the chains and dissociated ions have the same sizes $a$, so we can define the reduced density $\rho^{*}$ as follows

$$
\rho^{*}=a^{3}\left(\rho_{-}+\rho_{+}\right)
$$

The second term on the right hand side of Eq. (2) is the electrostatic contribution to the free energy. Using RPA approach we write it in the form ${ }^{5}$

$$
\begin{array}{r}
\frac{F_{\mathrm{el}}}{T V}=\frac{1}{4 \pi^{2}} \int_{0}^{\mathcal{K}}\left[\ln \left(1+\left(\rho_{+}+\rho_{-} g(k)\right) \frac{U(k)}{T}\right)-\right. \\
\left.\left(\rho_{+}+\rho_{-}\right) \frac{U(k)}{T}\right] k^{2} d k
\end{array}
$$

where the structure function of the chain $g(k)$ has the following definition

$$
g(k)=\frac{1}{N} \sum_{i, j}\left\langle e^{-i \mathbf{k}\left(\mathbf{r}_{i}-\mathbf{r}_{j}\right)}\right\rangle
$$

Indices $i$ and $j$ in Eq. (6) run over all monomers of the chain and the average $\langle\ldots\rangle$ is taken over all possible chain confirmations. $U(k)$ in Eq. (5) is the Fourier transform of the interaction potential between charges and the parameter $\mathcal{K}$ is used to cut the integration over the large values of $k$. The purpose of the present work is to discuss the RPA approximation, and determine appropriate forms of $U(k)$ and $\mathcal{K}$.

\section{INTERACTIONS BETWEEN CHARGES}

For dilute solutions of electrolytes the potential of interaction between charges can be taken in the form

$$
U(r)=\frac{q^{2}}{r \varepsilon}
$$

where $r$ is the distance between charges and $\varepsilon$ is the dielectric constant of the media. With this potential Eq. (5) calculated for a simple case of $N=1$ and $\mathcal{K}=\infty$ reduces to $-\kappa^{3} / 12 \pi$. That is, the electrostatic contribution reduces to the limiting case $(\kappa a \ll 1)$ of the $\mathrm{DH}$ expression for the free energy of hard-sphere ionic fluid,

$$
\frac{F_{\mathrm{el}}^{\mathrm{DH}}}{T V}=-\frac{\kappa^{3}}{12 \pi} \tau^{\mathrm{DH}}(\kappa a)
$$

Here the inverse screening length $\kappa$ is given by $\kappa^{2}=$ $4 \pi q^{2} l_{B}\left(\rho_{+}+\rho_{-}\right)$, the Bjerrum lenght $l_{B}=e^{2} / \varepsilon T$ and

$$
\tau^{\mathrm{DH}}(x)=\frac{3}{x^{3}}\left(\ln (1+x)-x+\frac{x^{2}}{2}\right)
$$

Clearly, Eq. (7) for $U(r)$ can not be used in the RPA approach if $\kappa a \sim 1$. As the value of $\kappa a$ increases the hardsphere interactions between the charges become more and more important and they should be taken into account. For this purpose we introduce the following form of $U(r)$

$$
U(r)=\frac{q^{2}}{r \varepsilon}(1-\exp (r / a))
$$

which suppresses electrostatic interactions on the scale of the size of the monomer. The Fourier transform of the above potential can be easily calculated

$$
U(k)=\frac{4 \pi}{k^{2}\left(1+k^{2} a^{2}\right)}
$$

and for the electrostatic free energy given by Eq. (5) we get

$$
\begin{array}{r}
\frac{F_{\mathrm{el}}}{T V}=\frac{1}{4 \pi^{2}} \int_{0}^{\mathcal{K}}\left[\ln \left(1+\frac{\kappa^{2}(1+g(k))}{2 k^{2}\left(1+k^{2} a^{2}\right)}\right)-\right. \\
\left.\frac{\kappa^{2}}{k^{2}\left(1+k^{2} a^{2}\right)}\right] k^{2} d k
\end{array}
$$

The integral in Eq. (12) can be done analytically for the case of $N=1$ and $\mathcal{K}=\infty$ which results in the following expression for the free energy

$$
\frac{F_{\mathrm{el}}^{\infty}}{T V}=-\frac{\kappa^{3}}{12 \pi} \tau^{\infty}(\kappa a)
$$

where

$$
\tau^{\infty}(x)=\frac{1}{x^{3}}\left(-1+\frac{3 x^{2}}{2}+\frac{1+x-2 x^{2}}{(1+2 x)^{1 / 2}}\right)
$$


Another way to account for the hard sphere nature of the charges is to use the potential (7) but cut integration in $(5)$ at $\mathcal{K}=1 / a$. This results in the following form of the free energy

$$
\frac{F_{\mathrm{el}}^{\mathcal{K}}}{T V}=-\frac{\kappa^{3}}{12 \pi} \tau^{\mathcal{K}}(\kappa a)
$$

where

$$
\tau^{\mathcal{K}}(x)=\frac{1}{\pi}\left(2 \arctan \left(x^{-1}\right)-\frac{\ln \left(1+x^{2}\right)-x^{2}}{x^{3}}\right)
$$

In Figure 1 we compare the results for $\tau^{\mathrm{DH}}(x), \tau^{\infty}(x)$, and $\tau^{\mathcal{K}}(x)$, given by Eqs. (9), (14), and (16) respectively. The curves presented in the figure show similar behavior as parameter $x=\kappa a$ increases. In Figure 2 we also show the phase diagrams obtained on the plane $\left(\rho^{*}, T^{*}=a / l_{B}\right)$ using different form of the electrostatic free energy given by Eqs. (8), (13), and (15). The figure clearly shows that the phase diagram obtained using Eq. (13) is in reasonable agreement with Debye-Hückel theory where as the one obtained using Eq. (15) differs significantly. This fact leads us to the conclusion that the hard-core interactions between the charges could be correctly taken into account through the potential $U(r)$ given by Eq. (10), but not through the large $k$ cut-off integration parameter $\mathcal{K}=1 / a$. However, in the next section we show that a different form of the cut-off $\mathcal{K}$ is very important for the proper discription of polyelectrolyte solutions.

\section{POLYELECTROLYTE SOLUTIONS}

We first examine the dilute case of infinitely long polyelectrolyte chains. In order to calculate the integral (5) analytically we assume that the macromolecules obey Gaussian statistics. For $g(k)$ defined by Eq. (6) we use the following approximation

$$
g(k)=1+\frac{N}{1+N b^{2} k^{2} / 12} \simeq 1+\frac{12}{b^{2} k^{2}}
$$

where $b^{2}$ is the mean-squared distance between the neighboring monomers of the chain. For simplicity we assume here that $b=a$. We also use $U(r)$ in the form (7) valid for the dilute case $\kappa a \ll 1$ and we rewrite Eq. (12) with $\mathcal{K}=\infty$ in the form

$$
\frac{F_{\mathrm{el}}}{T V}=\frac{1}{4 \pi^{2}} \int_{0}^{\infty}\left[\ln \left(1+\frac{\kappa^{2}}{k^{2}}+\frac{6 \kappa^{2}}{a^{2} k^{4}}\right)-\frac{\kappa^{2}}{k^{2}}\right] k^{2} d k
$$

Evaluation of the above integral leads to

$$
\frac{F_{\mathrm{el}}}{T V}=\frac{\kappa^{3 / 2}}{12 \pi a^{3 / 2}} \tau(\kappa a)
$$

where

$$
\tau(x)=\frac{12-\sqrt{6} x-x^{2}}{(\sqrt{24}+x)^{1 / 2}}
$$

The result $F_{\text {el }} \sim \kappa^{3 / 2}$ for infinitely long Gaussian chains was previously obtained in a number of papers (for example see Refs. 5, 17).

With the use of Eq. (19) we take the second derivative of the free energy (2) with respect to the density $\rho=$ $\rho_{+}+\rho_{-}$and in the limit of $\rho \rightarrow 0$ we find

$$
\frac{\partial^{2}}{\partial \rho^{2}}\left(\frac{F_{\mathrm{el}}}{T V}\right) \simeq \frac{1}{2 \rho}-\frac{\kappa^{3 / 2}}{64 \pi b^{3 / 2} \rho^{2}}<0
$$

The negative sign of the second derivative of the free energy shows that dilute solution of infinitely long polyelectrolyte chains are unstable in good solvent conditions. ${ }^{7}$ In Figure 3 we show the phase diagrams on the plane $\left(\rho^{*}, T^{*}=a / l_{B}\right)$ obtained for different chain lengths $N$. The electrostatic contribution to the free energy used to obtain the diagrams is

$$
\begin{aligned}
\frac{F_{\mathrm{el}}}{T V}=\frac{1}{4 \pi^{2} a^{3}} \int_{0}^{\infty} & {\left[\ln \left(1+\frac{(\kappa a)^{2}\left(3 / 2+N k^{2} / 12\right)}{k^{2}\left(1+k^{2}\right)\left(1+N k^{2} / 12\right)}\right)-\right.} \\
& \left.\frac{(\kappa a)^{2}}{k^{2}\left(1+k^{2}\right)}\right] k^{2} d k
\end{aligned}
$$

and it is evaluated numerically. The figure clearly demonstrates that as $N$ increases the region of instability becomes broader and broader even at very dilute concentrations. The same results can be obtain if we introduce a constant cut-of value $\mathcal{K}^{*}=\mathcal{K} a=2 \pi$.

We suggest here that the unphysical behavior described above arises from the inappropriate contribution to the RPA electrostatic free energy at large $k$. At large $k$ values the chains are perturbed due to the electrostatic interactions. RPA, however, is only valid if the interactions do not modify the chain conformations. Therefore, the cut-off has to be chosen such that we only include the electrostatic contributions that can be accounted by RPA. It is straight forward to determine the cut-off given that the major feature of polyelectrolyte solutions is the existence of a concentration dependent correlation length $\xi .{ }^{10}$ On the scales smaller then $\xi$ the chain is stretched and on larger scales it obeys Gaussian statistics. For strongly charged polyelectrolytes $\xi$ can be estimated as ${ }^{11,12}$

$$
\xi=\frac{1}{\left(\rho^{*}\right)^{1 / 2}}
$$

In Fourier space scales smaller than $\xi$ are given by $k a>$ $2 \pi / \xi$. Since the contributions to the free energy on these scales can not be accounted within the RPA approach, we cut integral (22)

$$
\mathcal{K}^{*}=2 \pi\left(\rho^{*}\right)^{1 / 2}
$$




\section{RESULTS, DISCUSSION AND CONCLUSIONS}

In Figure 4 we show the modified RPA phase diagrams obtained by evaluating the integral (22) up to $k a=\mathcal{K}^{*}$ with $\mathcal{K}^{*}$ given in (24). The modified RPA phase diagram has a much lower value of the critical temperature for large $N$ than the standard RPA phase diagram Figure 3. The critical temperature initially increases as $N$ increases in the modified RPA phase diagram, and it plateaus at very high $N$ values at about $l_{B} / b \sim 7.3$. Since most polymers have values of $l_{B} / b<4.2$ at room temperature, salt-free solutions of polyelectrolytes with monovalent counterions are water soluble at room temperatures in agreement with the experiments.

Another important feature of the modified RPA phase diagram in Figure 4 is the prediction that the critical concentration remains nearly constant as $N$ increases in agreement with the simulations. This is only true if the contributions from fluctuations of $k>\mathcal{K}$ are completely ignored. That is, if we add the RPA electrostatic contribution from the $2 \pi / a>k>\mathcal{K}$ in the free energy using the structure function for rod like units, though the critical temperature only increases slightly, the critical concentration goes to zero as $N$ increases. This suggests that the charge fluctuations at lower length scales do not contribute to the free energy or that they are strongly suppressed. The suppression of charge fluctuations on shorter length scales that $\xi$ can be explained by the fact that the electrostatic interactions are not screened at these length scales. Therefore, the counterions and monomers are strongly correlated. Indeed, these correlations stretched the chains.

We argue above that in polyelectrolyte solutions the cut-of is a natural inverse length over which RPA breaks down. It is important to point out, however, that a concentration dependent integration cut-off is also obtained in the one component plasma, ${ }^{18}$ in which counterions fluctuate over a non-fluctuating charged medium. The physical reason and the concentration dependence of the cut-off in the one component plasma explained in Ref. 18 is different than in polyelectrolytes. In a one component plasma the integration in the electrostatic free energy given by the RPA approach should be carried over a finite number of the wave-vectors $\mathbf{k}$ in the same way as in the Debye theory of the specific heat of solids. ${ }^{19}$ Namely, the total number of degrees of freedom in the system $3 \mathcal{N}=3 \rho V$ should be equal to the total number of physically different modes with wave-vectors $\mathbf{k}$ within the spherical shell of radius $\mathcal{K}$. The number of modes is twice the number of the wave-vectors since each $\mathbf{k}$ has a sine a cosine mode. Therefore, one obtains

$$
2 V \int_{0}^{\mathcal{K}} \frac{d^{3} k}{(2 \pi)^{3}}=3 \mathcal{N}
$$

which leads to

$$
\mathcal{K}^{*}=\left(9 \pi^{2} \rho\right)^{1 / 3}
$$

The above argument assumes that the important length scale is the average distance between ions $d \sim$ $2 \pi / \mathcal{K} \sim \rho^{-1 / 3}$, and that this length scales imposes a "periodicity" even though the non fluctuating support for the ions is a solid with no structure.

The distance between charges $d \sim 1 / \rho^{1 / 3}$ is an important length scale to determine the break down of DH in simple electrolyte solutions. ${ }^{20}$ Therefore, in simple electrolytes $d$ is an important length scale as the system gets concentrated when the electrostatic energy between two charges separated by $d, e^{2} /(4 \pi \varepsilon d)>K_{B} T$ or $l_{B} / d>1$. Interestingly, the integration to a concentration dependent cut-off $\mathcal{K} \sim \rho^{1 / 3}$ versus integrating to the inverse hard core size of the ions $\mathcal{K} \sim 1 / a$ does not affect significantly the RPA phase diagrams in simple electrolytes. Instead, in salt-free polyelectrolyte solutions a concentration dependent cut-off is essential to recover reasonable values of the critical temperature as $N$ increases and a $N$ independent critical concentration.

Though we argue that in salt-free polyelectrolyte solutions the natural length scale that determines the cut-off is $\xi$, we obtain very similar phase diagrams if we instead use $d$ as shown in Figure 5. Notice that the importance of a concentration dependent cut-off is at dilute solutions where $\xi>d$. Therefore, there should be counterion fluctuations at length scales $d<l<\xi$ that we did not include in our RPA approach. If we add a RPA contribution from these counterion fluctuations (only the counterions) we get negligible corrections to the phase diagram. Our arguments are in agreement with the results in Ref. 21 where a self-consistent polyelectrolyte RPA where the screening length from polyions is wavevector-dependent due to chain connectivity shows that at high wavevectors (short length scales), only counterions contribute to screening. At lower wavevectors, both polyions and counterions contribute to screening.

In summary, we show that in salt-free solutions a wave vector dependent cut-off is essential to recover physical phase diagrams. The cut-off is determined by the applicability limit of the RPA. In the presence of salt the electrostatic contribution to the free energy reduces to the well known DH limiting law. ${ }^{5}$ In that case reasonable phase diagram are obtain without a concentration dependent cut-off, ${ }^{22}$ although in principle one should only include fluctuations from the monomers on the length scale where the RPA approach is valid (i.e., larger than $\xi$ ).

We acknowledge the financial support of the NIH grant number GM62109-02 and of the Institute for Bioengineering and Nanosciences in Advanced Medicine (IBNAM) at Northwestern University.

\footnotetext{
${ }^{1}$ Conway, B. E. "Ionic Interactions and Activity Behavior of Electrolyte Solutions" in B. E. Conway, J. O’M. Bockris, E.
} 
Yeager: Comprehensive Treatise of Electrochemistry; v.5. New York: Plenum Press (1983).

${ }^{2}$ Orkoulas, G; Panagiotopoulos, A. Z. J. Chem. Phys. 1999, 110, 1581. Yan, Q.; De Pablo, J.J. J. Chem. Phys. 1999, 111, 9509. Luijten, E.; Panagiotopoulos, A. Z.; Fisher, M. E. Phys. Rev. Lett. 2002, 88, 045701.

${ }^{3}$ Bjerrum, N. Koninklinge Dans. Vidensk. Selsk. 1926 7, 9.

${ }^{4}$ Fisher, M. E.; Levin, Y. Phys. Rev. Lett. 1993, 71, 3826.

${ }^{5}$ Borue, V. Yu.; Erukhimovich, I. Ya. Macromolecules 1988, 21,3240 .

${ }^{6}$ Joanny, J. F.; Leibler, L. J. Phys. France, 1990, 51(6), 545.

${ }^{7}$ Mahdi, K. A.; Olvera de la Cruz, M. Macromolecules, 2000, 33, 7649 .

${ }^{8}$ Orkoulas G.; Kumar Sanat K. preprint 2002.

${ }^{9}$ M. Muthukumar; Macromolecules, 2002 35, 9142.

${ }^{10}$ de Gennes, P.G. Scaling Concepts in Polymer Physics; Cornell Univ. Press: 1979.

${ }^{11}$ Schiessel, H.; Pincus, P. Macromolecules 1998 31, 7953. Schiessel, H. ; Macromolecules, 1999, 32, 5673.

12 Dobrynin, A. V.; Rubinstein, M. Macromolecules, 1999, 32, 915.

13 Stevens M. J.; Kremer K. J. Chem. Phys. 1995, 103, 1669.

${ }^{14}$ U. Micka, C. Holm, K. Kremer Langmuir 1999, 15, 4033. Stevens, M.; Kremer, K. J. Phys. II France, 1997, 6, 1607.

${ }^{15}$ Manning G. S. J. Phys. Chem. 88 (1984) 6654

${ }^{16}$ P. Gonzales-Mozuelos, M. Olvera de la Cruz: J. Chem. Phys. 1995, 103, 3145.

${ }^{17}$ Olvera de la Cruz, M.; Belloni, L.; Delsanti, M.; Dalbiez, J.; Spalla, O.; Drifford, M. J. Chem. Phys., 1995, 103, 5781.

18 Brilliantov, N. V.; Malinin, V. V.; Netz, R. R. Eur. Phys. J. D 2002, 18, 339 .

${ }^{19}$ Ziman, J. M. Principles of the Theory of Solids; Claredon Press: Oxford, 1964.

${ }^{20}$ Brilliantov, N. V.; Kuznetsov, D. V; Klein, R. Phys. Rev. Lett., 1998, 81, 1433.

${ }^{21}$ Donley, J. P.; Rudnick, J.; Liu, A. J. Macromolecules, 1997, 30, 1188.

22 Mahdi,K. A. PhD thesis (Northwestern University, USA, 2000).

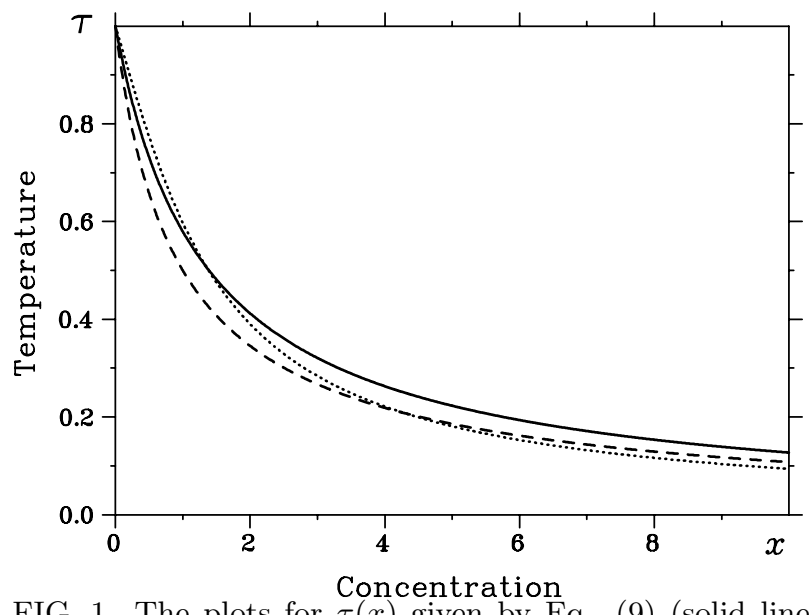

FIG. 1. The plots for $\tau(x)$ given by Eq. (9) (solid line), Eq. (14) (dash line), and Eq. (16) (dot line).

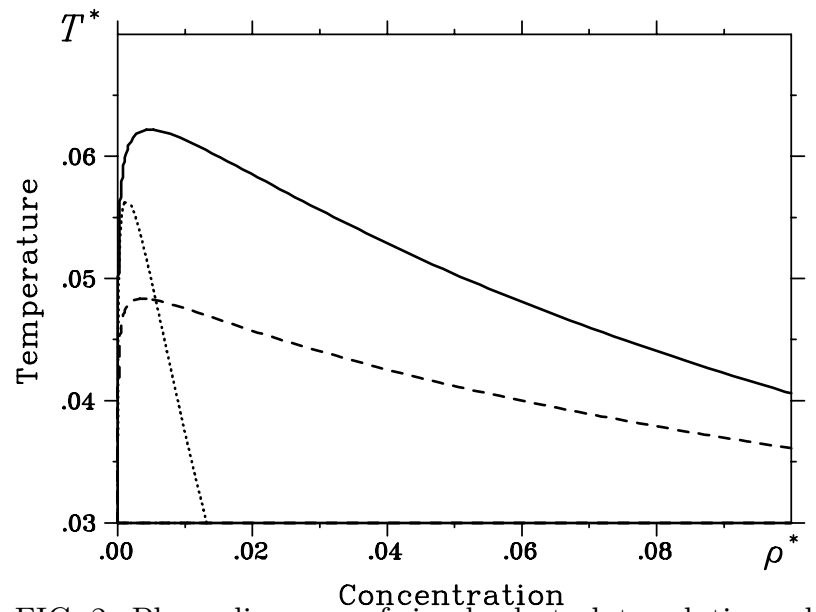

FIG. 2. Phase diagrams of simple electrolyte solutions obtained using different forms of electrostatic free energy given by Eq. (8) (solid line), Eq. (13) (dash line), and Eq. (15) (dot line).

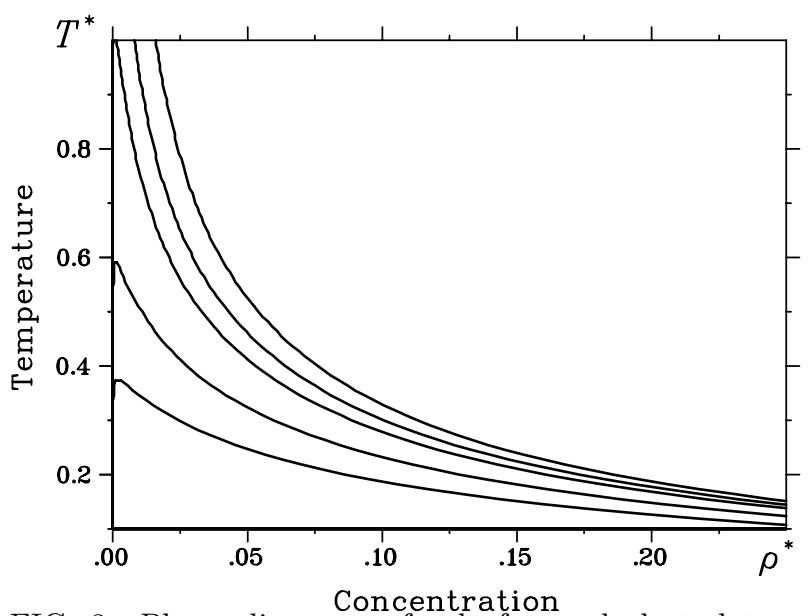

FIG. 3. Phase diagrams of salt free polyelectrolyte solutions obtained within classical RPA approach for different lengths of the polymers $N$. From buttom to top $N=10,20,50,100,1000$.

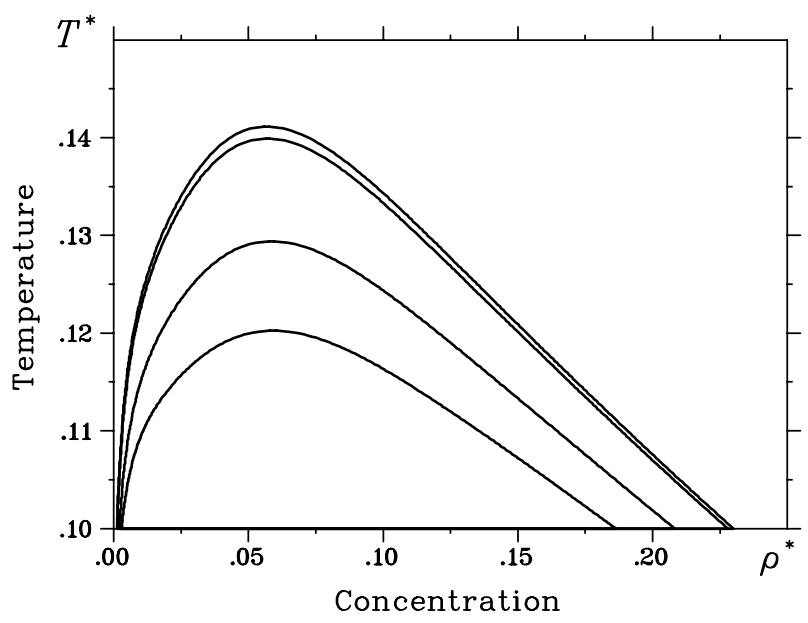


FIG. 4. Phase diagrams of salt free polyelectrolyte solutions obtained within modified RPA approach with $\mathcal{K}^{*}$ given by Eq. (24) for different lengths of the polymers $N$. From buttom to top $N=50,100,1000,10000$.

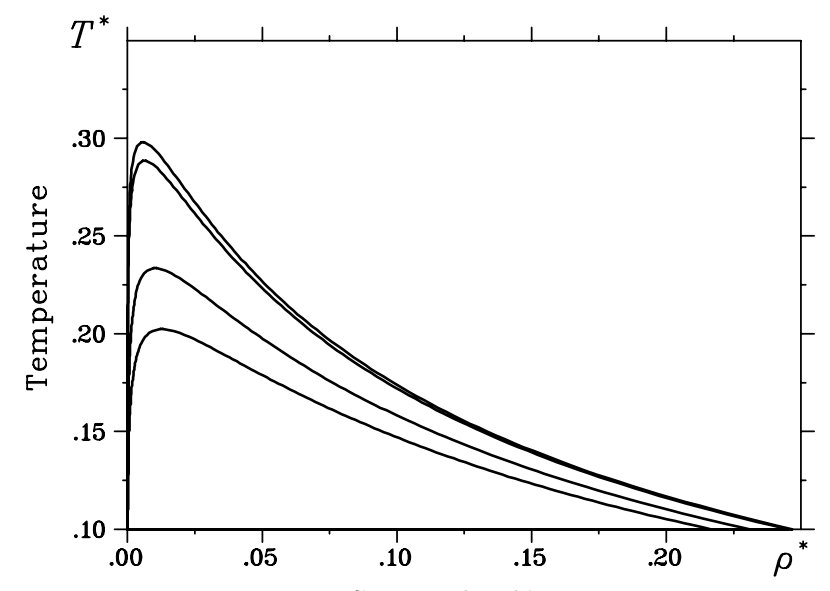

FIG. 5. Phase diagrams of salt free polyelectrolyte solutions obtained within modified RPA approach with $\mathcal{K}^{*}$ given by Eq. (26) for different lengths of the polymers $N$. From buttom to top $N=50,100,1000,10000$. 